Proceedings of the International Symposium on Physics of Materials (ISPMA 14), September 10-15, 2017, Prague

\title{
Profile Shape Effect on the Texture and Mechanical Properties of Extruded Rare Earth Containing Magnesium Alloys
}

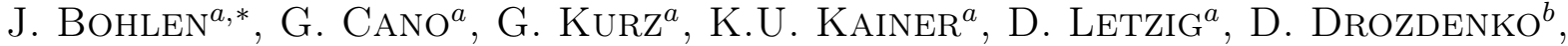 \\ F. CHMELIK $^{b}$ AND P. DOBRON ${ }^{b}$ \\ ${ }^{a}$ Magnesium Innovation Centre (MagIC), Helmholtz-Zentrum Geesthacht, \\ Max-Planck Str. 1, D 21502 Geesthacht, Germany \\ ${ }^{b}$ Department of Physics of Materials, Charles University, Ke Karlovu 5, CZ 12116 Prague 2, Czech Republic

\begin{abstract}
Magnesium alloys with rare earth or Ca additions often form weak textures during rolling of magnesium sheets or extrusion of round bars. However, in the case of the extrusion of flat profiles of such alloys strong textures may develop. This is of fundamental importance because weaker textures are associated with higher
\end{abstract} \\ ductility or formability. Variations in the activity of deformation and recrystallization mechanism play a role in \\ the microstructure and texture development during the underlying forming procedures. In this study, magnesium \\ alloys with $\mathrm{Zn}$ or $\mathrm{Mn}$ as the main alloying elements were modified with rare earth or Ca and extruded to flat profiles. \\ The microstructure and texture development is compared and related to the mechanical properties. Alloying and \\ processing aspects are discussed with a view to tailor the mechanical properties of such flat profiles.
}

DOI: 10.12693/APhysPolA.134.714

PACS/topics: 81.10.Jt, 81.20.Hy, 81.40.Lm, 81.70.Bt

\section{Introduction}

A general limitation of active deformation mechanisms and therefore ductility or formability of magnesium alloys is often associated with their hexagonal close packed (hcp) lattice structure. The directionality of such mechanisms also causes distinct anisotropic behavior, especially in textured magnesium alloys $[1,2]$. This drawback for wrought magnesium applications like sheets and profiles has been balanced by alloy and process selection to allow the production of semi-finished products with weak textures and therefore higher formability $[2-4]$. Several mechanism have been addressed to explain texture weakening including differences in the activation of deformation mechanisms [3, 5-7], especially different types of twins and shear bands $[8]$ as well as changes in the recrystallization behavior $[9,10]$. As a result, design concepts for mechanical properties and especially improved formability have been enabled. Alternative processing routes compared to the rolling of sheets have been emphasized in an effort to improve the production efficiency. This includes the extrusion of flat bands [11]. It has been shown that extrusion of magnesium alloy sheets even at high extrusion ratios is feasible.

Interestingly, there are fundamental differences in the resulting microstructures compared to sheet rolling. For example, the development of textures in flat extrusions appears significantly stronger compared to round bar counterparts. In Ref. [12] extruded sheets of alloys ZE10 and ME21 with rare earth (RE) additions revealed strong textures, whereas extruded round bars of the same or

*corresponding author; e-mail: jan.bohlen@hzg.de comparable alloys exhibit weak textures $[13,14]$. This latter aspect is addressed in the present work by comparing the texture development of flat extruded bands for modified $\mathrm{Mg}-\mathrm{Mn}$ - and $\mathrm{Mg}-\mathrm{Zn}$-based alloys. Additional alloying elements include $\mathrm{RE}$ and $\mathrm{Ca}$, which are known to act as texture modifiers during forming and recrystallization of magnesium alloys $[3,8]$.

\section{Experimental}

$\mathrm{Mg}-\mathrm{Zn}$ - and $\mathrm{Mg}-\mathrm{Mn}$-based alloys were used in this study for the extrusion of a flat bar. The alloys were modified by additional alloying with RE (Ce-mischmetal, $\mathrm{Nd}$ and $\mathrm{Gd}$ ) as well as Ca. The chemical composition is shown in Table I.

Billets for extrusion were produced by gravity casting followed by directional solidification and machined to the samples with a diameter of $49 \mathrm{~mm}$ and a length of $150 \mathrm{~mm}$. The billets were homogenized for $16 \mathrm{~h}$ before extrusion. The Mg-Mn-based alloys did not exhibit the possibility for solid solution annealing and were homogenized at $450^{\circ} \mathrm{C}$. In case of the $\mathrm{Mg}-\mathrm{Zn}$-based alloys more specific annealing conditions were selected. In case of Z1 and ZX10 solid solution annealing was carried out at $400{ }^{\circ} \mathrm{C}$ whereas it was at $500^{\circ} \mathrm{C}$ for alloys ZN10 and ZG02. Direct extrusion was carried out to produce flat bars using a 2.5 MN automatic extrusion press of Müller Engineering (Germany). The width of the profile was $20 \mathrm{~mm}$ and $2 \mathrm{~mm}$ in thickness, which corresponds to an extrusion ratio of 1:49. The $\mathrm{Mg}-\mathrm{Mn}$-based alloys were extruded at $300^{\circ} \mathrm{C}$ and an extrusion speed of $1.75 \mathrm{~mm} / \mathrm{s}$ whereas the $\mathrm{Mg}-\mathrm{Zn}$-based alloys were extruded at higher temperature to maintain solid solution (i.e. $400^{\circ} \mathrm{C}$ in case of Z1 and ZX10 and $450^{\circ} \mathrm{C}$ in case of ZN10 and ZG02) and an extrusion speed of $2.4 \mathrm{~mm} / \mathrm{s}$. 
Chemical composition of the alloys in

TABLE I this study; values in wt\%; $\mathrm{Mg}$ balance

\begin{tabular}{c|c|c|c|c|c|c|c|c}
\hline \hline Alloy & Mn & Zn & Ce & Nd & La & Pr & Gd & Ca \\
\hline ME20 & 1.74 & - & 0.21 & 0.046 & 0.13 & 0.016 & - & - \\
ME21 & 1.83 & - & 0.49 & 0.128 & 0.31 & 0.038 & - & - \\
MN20 & 2.01 & - & - & 0.48 & - & - & - & - \\
MX21 & 1.87 & - & - & - & - & - & - & 1.04 \\
Z1 & - & 0.91 & - & - & - & - & - & - \\
ZN10 & - & 0.98 & - & 0.57 & - & - & - & - \\
ZG02 & - & 0.51 & - & - & - & - & 1.97 & - \\
ZX10 & - & 0.94 & - & - & - & - & - & 0.15
\end{tabular}

Standard metallography procedures on longitudinal sections by using an etchant based on picric acid [15] were applied to reveal the grain structure. The texture was measured on the surface of the extrusions after grinding and polishing. A Panalytical X-ray diffractometer with $\mathrm{Cu} K_{\alpha}$ radiation was used to measure pole figures. Recalculation of full pole figures was carried out using the open-source computer code MTEX [16].

Orientation imaging was performed on longitudinal sections using electron backscatter diffraction (EBSD) in a field emission gun scanning electron microscope (Zeiss Ultra 55, EDAX/TSL EBSD system and Hikari detector). After using metallography procedures to prepare the surface, electropolishing was applied using an AC2 solution $\left(\right.$ Struers $\left.^{T M}\right)$. A software "TSL Orientation Imaging Microscopy Analysis" of EDAX(C)was used to analyse the measurements and visualise specific fractions of the microstructure. This includes separation of grain fractions
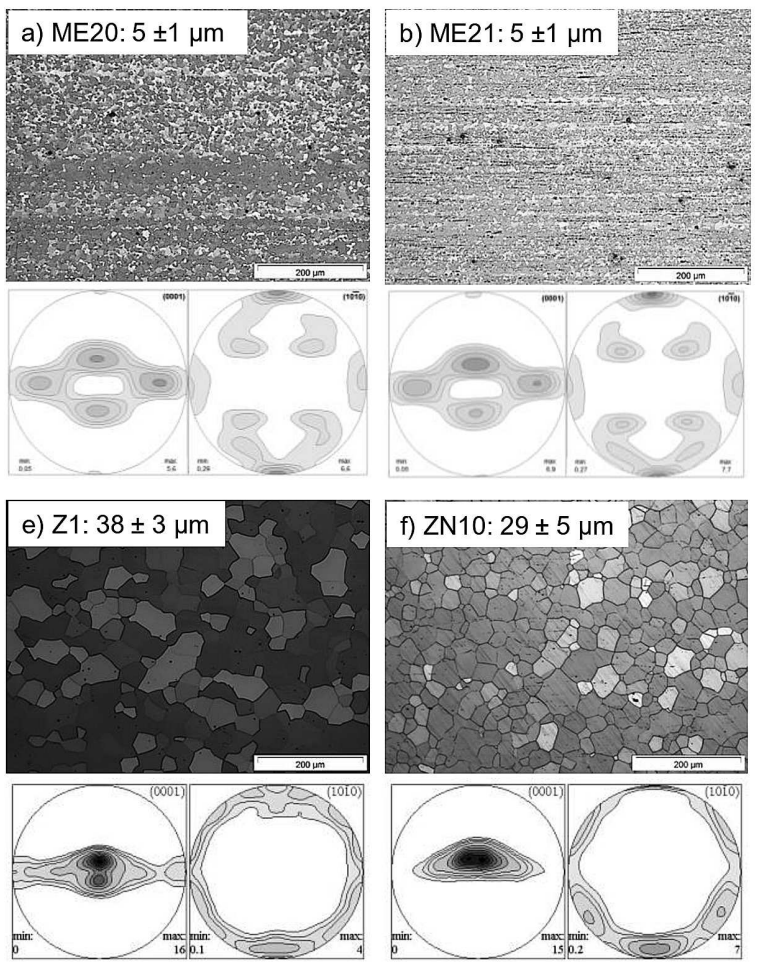

along with a function to separate grains with different grain orientation spread (GOS). In this approach an arbitrary limit of $1^{\circ}$ is used as a separator, assuming that grains with low orientation spread can be understood as recrystallised grains [13].

Mechanical properties were evaluated by tensile tests on a universal testing machine Zwick Z050 at room temperature and a constant initial strain rate of $1.0 \times 10^{-3} / \mathrm{s}$. Tensile samples with a gauge length of $18 \mathrm{~mm}$ were prepared by using spark erosion and tested parallel to the extrusion direction.

\section{Results}

Figure 1 shows the resulting micrographs and pole figures of the extruded bands after extrusion. In case of the $\mathrm{Mg}-\mathrm{Mn}$-based alloys (Fig. $1 \mathrm{a}-\mathrm{d}$ ) the variations in the grain structure appear small. Both, ME20 and ME21 exhibit a structure with non-recrystallized grains elongated parallel to extrusion direction as well as particle stringers. Particle stringers are associated with particles from the original cast billet structure that also underwent deformation, i.e. they did not form during extrusion. This effect is less visible in case of MN20, revealing a fully recrystallized grain structure and a fine distribution of particles. The average grain size is comparable at $5 \mu \mathrm{m}$ in these alloys. In case of the Ca containing alloy MX21 again particle stringers are visible together with larger particles distributed in the microstructure. The grain size is only slightly larger at $7 \mu \mathrm{m}$, indicating a small change in the dynamic recrystallization (DRX) behavior.
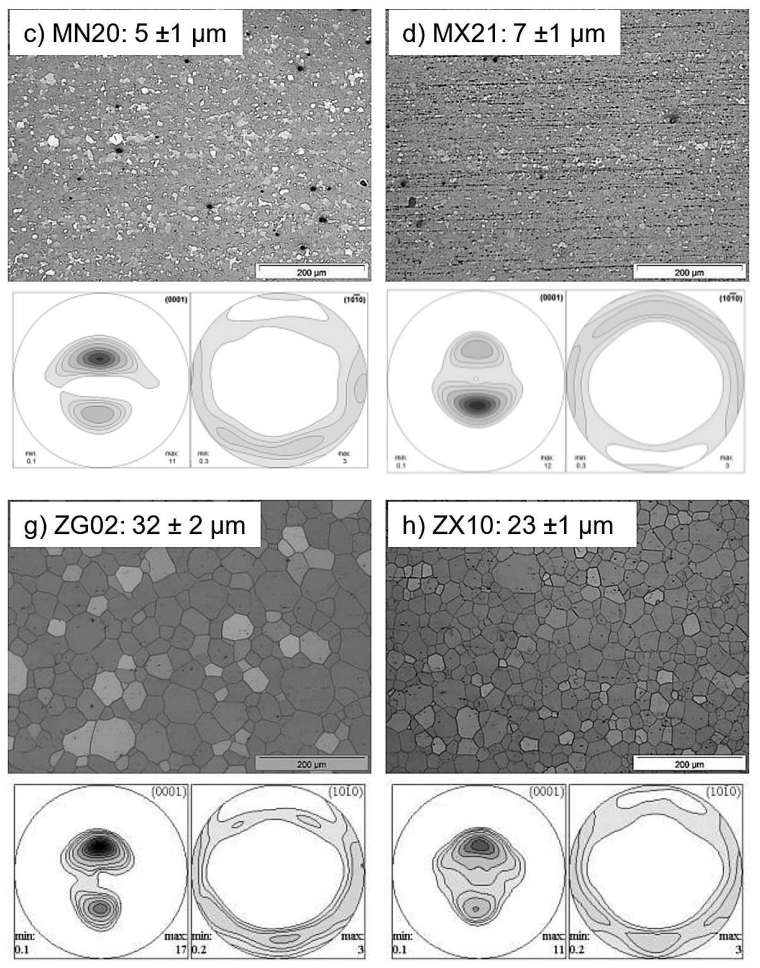

Fig. 1. Micrographs from longitudinal sections and pole figures from cross-sections of the alloys of this study (micrograph: ED horizontal, pole figures: ED vertical). 
The corresponding textures of MN20 and MX21 reveal pronounced split peaks of basal planes towards the extrusion direction (ED) and prismatic planes with a tilt out of the normal direction (ND). However, in ME20 and ME21 high prismatic pole intensities in ED are observed. A further component puts prismatic planes also parallel to the extrusion direction. Correspondingly, the basal planes are aligned in four weaker peaks, two of them being split to ED and two of them with a tilt towards the transverse direction (TD). Notably, this texture is unusual for flat extrusions [11] but reveals the classical prismatic fibre component often reported in magnesium extrusions [17].

The $\mathrm{Mg}-\mathrm{Zn}$-based alloys were extruded at higher temperatures as well as higher speed in an effort to maintain solid solution. Thus, the resulting microstructures in Fig. 1e-h appear coarser grained associated with enhanced DRX and/or grain growth. The largest grain size of $38 \mu \mathrm{m}$ in a fully recrystallized microstructure was obtained in alloy Z1. Additional alloying elements lead to finer-grained microstructures like in ZN10 with Nd and in ZX10 with Ca. A distinct reduction of the grain size due to $\mathrm{Ca}$ addition can be confirmed unlike in case of the Mg-Mn-based alloys. Alloy ZG02 contains a lower amount of $\mathrm{Zn}$ but a high amount of $\mathrm{Gd}$, however, grain refinement compared to the binary $\mathrm{Z} 1$ alloy is not very pronounced. Again, the textures of the alloys are varying quite significantly. In case of Z1 and ZN10 a weak prismatic fibre is found with a strong alignment of basal planes parallel to the surface of the band, but also with a broad angular spread towards TD. In case of ZG02 as well as in ZX10 two strong split peaks of basal planes with tilt towards ED are found. No prismatic alignment is identified which is comparable to the textures of MN20 and MX21. The same additional alloying elements do not necessarily maintain the same texture development if the main alloying element is varied from $\mathrm{Mn}$ to $\mathrm{Zn}$.

Figure 2 shows two example EBSD orientation maps of alloys ME20 and MN20 in order to visualize the source of different texture components. The pole figures labelled "all data" which uses the full measurement to reveal the pole figures leads to texture results comparable to the same alloys in Fig. 1. Two further pole figures are shown where only grains with specific GOS are used: with GOS $<1^{\circ}$ and GOS $>1^{\circ}$, respectively. Following an approach in Ref. [13] it is assumed that grains with low GOS mainly correspond with recrystallized grains which do not exhibit broad orientation distributions. On the contrary, grains with high GOS exhibit orientation variations which are consistent with active glide systems. The pole figure presentation in Fig. 2a for ME20 shows that unrecrystallized grains with GOS $>1^{\circ}$ have preferred orientation following the prismatic fibre as well a tilt of basal planes towards TD rather than those with tilt to ED. In case of the recrystallized grains with GOS $<1^{\circ}$ similarity to the overall texture is found, indicating that the orientation of deformed grains may be maintained during recrystallization but a further component with ED split is revealed. In case of MN20 in Fig. 2b an almost fully recrystallized microstructure is revealed where only a few unrecrystallized grains with GOS $>1^{\circ}$ exhibit orientations with tilt towards TD. However, this component is not well represented in the pole figures due to its low fraction. In summary, the TD split of basal planes can be associated especially with unrecrystallized grains and the alloys differ to maintain this component during recrystallization which is associated with the formation of the ED split of basal planes.
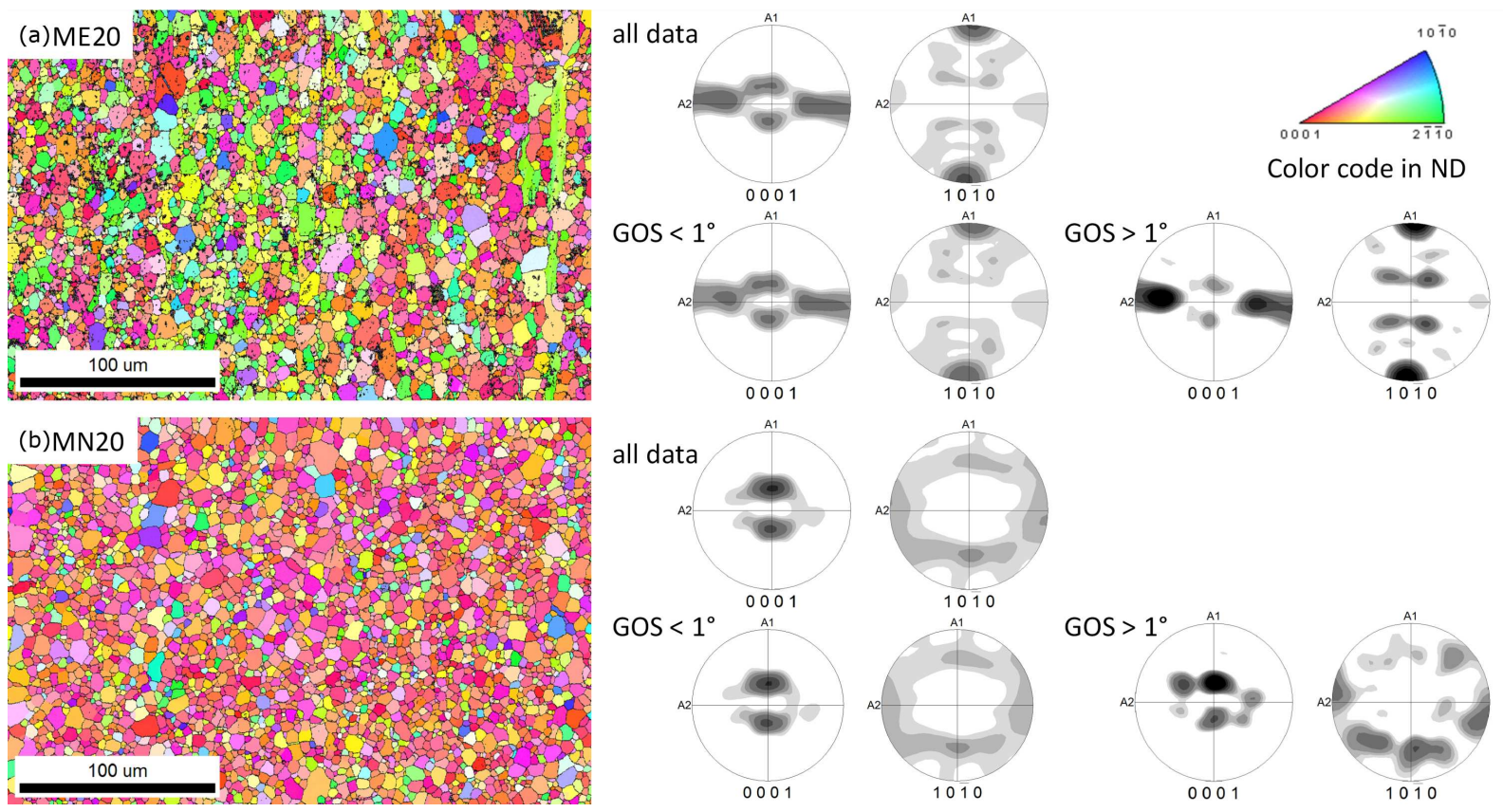

Fig. 2. EBSD orientation maps of (a) ME20 and (b) MN20; maps: ED vertical, ND horizontal; pole figures compare Fig. 1. 

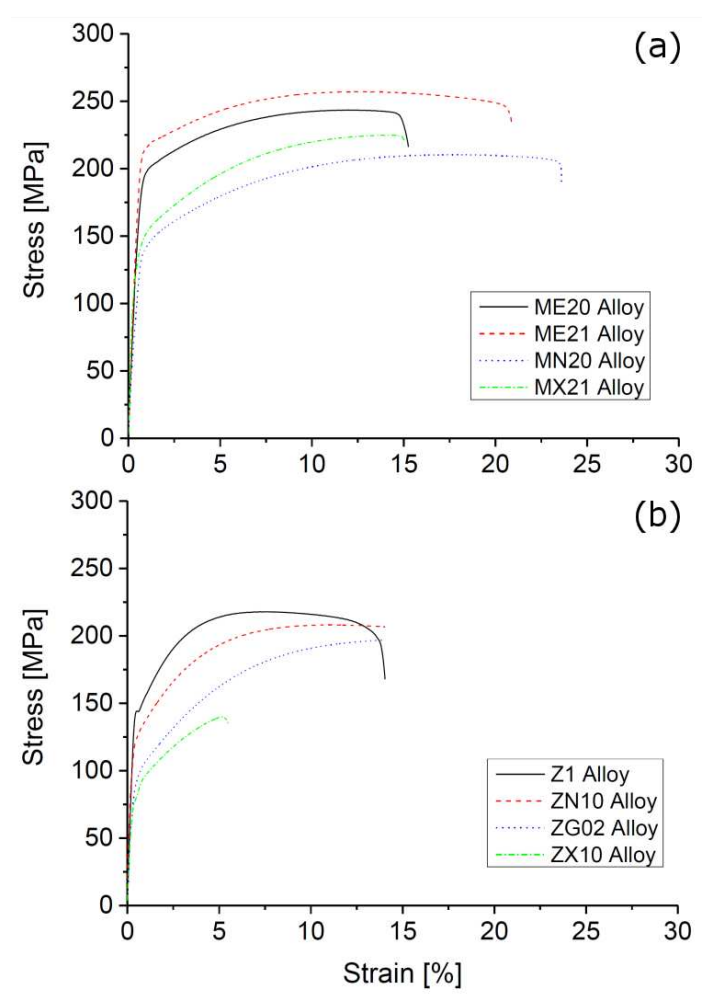

Fig. 3. Stress-strain diagrams from tensile tests parallel to ED: (a) Mg-Mn-based alloys, (b) Mg-Zn-based alloys.

TABLE II

Mechanical properties from tension tests parallel to ED (tensile yield stress (TYS), ultimate tensile stress (UTS)) with standard deviations

\begin{tabular}{c|c|c|c|c}
\hline \hline Alloy & $\begin{array}{c}\text { TYS } \\
{[\mathrm{MPa}]}\end{array}$ & $\begin{array}{c}\text { UTS } \\
{[\mathrm{MPa}]}\end{array}$ & $\begin{array}{c}\text { Uniform } \\
\text { elongation [\%] }\end{array}$ & $\begin{array}{c}\text { Fracture } \\
\text { strain [\%] }\end{array}$ \\
\hline ME20 & $164 \pm 7$ & $245 \pm 4$ & $11.3 \pm 1$ & $14.8 \pm 2.0$ \\
ME21 & $203 \pm 5$ & $256 \pm 2$ & $11.6 \pm 0.2$ & $20 \pm 1$ \\
MN20 & $131 \pm 26$ & $213 \pm 11$ & $16 \pm 1$ & $23 \pm 1$ \\
MX21 & $125 \pm 1$ & $223 \pm 2$ & $13.4 \pm 0.5$ & $14 \pm 0.4$ \\
Z1 & $144 \pm 1$ & $217 \pm 1$ & $6.8 \pm 0.3$ & $13 \pm 0.6$ \\
ZN10 & $116 \pm 4$ & $210 \pm 2$ & $11.1 \pm 0.7$ & $17.2 \pm 3.8$ \\
ZG02 & $89 \pm 5$ & $198 \pm 1$ & $16.0 \pm 0.3$ & $24.0 \pm 1.7$ \\
ZX10 & $75 \pm 2.3$ & $139 \pm 8$ & $4.8 \pm 0.2$ & $5.1 \pm 0.4$
\end{tabular}

Sample stress-strain curves from tensile tests parallel to ED are shown in Fig. 3. Generally, a continuous elastoplastic transition is followed by continuous work hardening to varying extent until the maximum stress (UTS) is reached followed by a flow instability which is associated with necking until the fracture of the samples. The beginning of instable flow is not reached in the Cacontaining alloys MX21 and ZX10, indicating a reduction in the ductility of such alloys. In Z1 a pronounced yield point is noted.

Mechanical properties are collected in Table II. Despite the quite similar grain sizes of the $\mathrm{Mg}-\mathrm{Mn}$-based alloys, distinct differences are observed. Lowest yield stresses (TYS) are found in MN20 and MX21 combined with extended work hardening to high UTS and high uniform elongation (15-16\%). Fracture occurs upon this maximum strain in MX21 whereas in MN20 the highest fracture strain in the $\mathrm{Mg}-\mathrm{Mn}$-based alloys is found. In comparison, ME20 and ME21 exhibit higher stress properties (TYS and UTS). However, the increase of stress to UTS is not pronounced indicating limited work hardening and resulting in lower uniform elongation. Interestingly, the higher RE containing alloy ME21 exhibits not only higher fracture strain but also higher TYS. The principal difference in mechanical properties between the $\mathrm{Mg}-\mathrm{Mn}$-based alloys is associated with the different texture of the samples.

Likewise, in case of the $\mathrm{Mg}-\mathrm{Zn}$-based alloys, those with a strong split peak of basal planes, ZX10 and ZG02, exhibit the lowest TYS, enhanced work hardening and in case of ZG02 highest uniform elongation as well as fracture strain. Z1 and ZN10 with a different texture and a preferential alignment of basal planes parallel to ED exhibit higher TYS, lower work hardening, lower uniform strain and lower fracture strain. UTS for Z1 and ZN10 is highest among the alloys of this $\mathrm{Mg}-\mathrm{Zn}$ series.

\section{Discussion}

In extruded round bars of magnesium alloys several developing texture components have been identified. Classically, a prismatic fibre texture with a prismatic $\langle 10.0\rangle$ pole parallel to ED is described [17]. Recrystallized microstructures of such bars often tend to develop a tilt component up to $30^{\circ}$, putting the intensity distribution along an arc between the $\langle 10.0\rangle$ and $\langle 11.0\rangle$ poles or even closer to the $\langle 11.0\rangle$ pole $[18,19]$. The result of both textures is a distinct alignment of basal planes parallel to $\mathrm{ED}$ or the $c$-axis normal to the profile surface. In RE containing alloys $[8,13]$ textures with intensities tilting a possible $\langle 10.0\rangle$ and $\langle 11.0\rangle$ - double fibre towards $\langle 00.1\rangle$ have been observed which results in a tilt of basal planes related to ED. These three texture components can be used to analyze the texture of the flat bands of this work.

Binary alloy Z1 without additional RE or Ca additions exhibits a distinct alignment of basal planes parallel to the profile surface and with an angular spread towards TD. This corresponds to a prismatic fibre component and the above mentioned classical texture development during extrusion. The Nd containing alloy ZN10, however, does not show any distinct variation from this texture which is somewhat unexpected for a RE containing alloy. It can be hypothesized that high extrusion temperatures still can change preferential growth of grains and that the formation of the rare earth texture can be overcome [8].

In case of RE or Ca containing MN20 and MX21 no such prismatic fibre is visible but a small tilt of prismatic planes towards ND, very similar to the above mention RE texture component. A corresponding split peak of basal planes towards ED is comparable to the tilt of basal planes out of the extrusion direction in round bars, i.e. a tilt of basal planes out of the surface plane of the 
profile [11]. Thus, the strong split peak texture of these flat bands is associated with the RE-texture which is observed in fully recrystallized profiles. The same is found for alloys ZG02 and ZX10. It can be noted that these alloys can be kept with the alloying elements in solid solution which is not possible in MN20 and MX21. Thus, the formation of this corresponding texture is not necessarily associated with any growth restriction of grains during recrystallization due to the formation of precipitates [20].

For ME20 and ME21 a prismatic fibre texture component is found. This corresponds to the partly recrystallized microstructures in Fig. 1. This fibre-texture can often be associated with the unrecrystallized fraction of grains [13], which is confirmed in Fig. 2. The corresponding split peaks towards TD therefore also indicate the orientations of unrecrystallized grains. This varies from the findings in Z1 and ZN10 with an alignment of basal planes parallel to the profile surface. It is noteworthy that such specific resolution of texture components (i.e. the split peak towards TD) would be hindered in round bars due to the rotational symmetry of the profile. However, a second component with basal planes split towards $\mathrm{ED}$ is also found indicating the RE-type texture development during recrystallization.

In summary, both a classical texture development and a rare earth type texture development are identified in flat extruded bands. A more complex texture with multiple texture components in ME20 and ME21 is associated with a not fully recrystallized microstructure but a RE-type texture development.

A tilt of basal planes out of a main deformation direction allows easier activation of basal slip, most likely the most influencing mechanism for the increase of work hardening during mechanical testing as well as for the increase of ductility and formability [3]. Unfortunately, such textures can also be associated with pronounced anisotropic behavior [12]. Although the aspect of anisotropic behavior cannot be revealed in measurements in this study, this may be less significant in the both other textures, alignment of basal planes parallel to the profile surface as well as in the case of the 4 split peak texture of ME20 or ME21. It is however revealed that in the ME-alloys slightly lower uniform elongation is accompanied by higher stress properties compared to the strong single split peak texture. Differences between the stress properties of the $\mathrm{Mg}-\mathrm{Mn}$-based alloys and the $\mathrm{Mg}-$ Zn-based alloys of this study are related to the significant differences in the grain size.

\section{Summary and conclusions}

The texture development of round bar extruded profiles and flat band extruded profiles shows similarities with respect to the relative alignment of basal planes in relation to the profile surface. Classical prismatic fibre textures lead to an alignment of basal planes parallel to the band surface whereas a RE-type texture with tilt of basal planes reveals strong split peaks but no prismatic fibre. Other textures with distinct components are observed in partly recrystallized samples. The related mechanical properties underline the importance of orientations for favoured basal slip which is related to lower yield stresses but higher work hardening ability, higher uniform elongation and fracture strain. Although Ca containing alloys lead to similar texture development like RE containing alloys as well as to corresponding mechanical behaviour, the ductility of these alloys is lower and fracture occurs early. A balancing of the texture development for controlling mechanical properties can be envisioned by acknowledging an ensemble of alloying as well as processing effects on the microstructure and texture development.

\section{Acknowledgments}

The authors appreciate financial support of this work which is a part of Grant No. 17-21855S by the Czech Science Foundation (GACR). Author G.C. also acknowledges financial support for his stay at the HelmholtzZentrum Geesthacht by the National Council of Science and Technology of Mexico (CONACyT) and the German Academic Exchange Service (DAAD). The authors would like to thank Mr. Günter Meister for his help in alloy casting and Mr. Alexander Reichart for billet machining as well as Mrs. Petra Fischer for her help during EBSD measurements.

\section{References}

[1] J. Bohlen, G. Kurz, S. Yi, D. Letzig, in: Advances in Wrought Magnesium Alloys: Fundamentals of Processing, Properties and Applications, Eds. C.J. Bettles, M.R. Barnett, Woodhead Publishing, Cambridge (UK) 2012, p. 346

[2] A.G. Beer, in: Advances in Wrought Magnesium Alloys: Fundamentals of Processing, Properties and Applications, Eds. C.J. Bettles, M.R. Barnett, Woodhead Publishing, Cambridge (UK) 2012, p. 304.

[3] J. Bohlen, M.R. Nürnberg, J.W. Senn, D. Letzig, S.R. Agnew, Acta Materialia 55, 2101 (2007).

[4] L. Stutz, J. Bohlen, G. Kurz, D. Letzig, K.U. Kainer, Key Eng. Mater. 473, 335 (2011).

[5] A. Styczynski, C. Hartig, J. Bohlen, D. Letzig, Scr. Mater. 50, 943 (2004).

[6] S.R. Agnew, M.H. Yoo, C.N. Tomé, Acta Mater. 49, 4277 (2001).

[7] J.P. Hadorn, K. Hantzsche, S. Yi, J. Bohlen, D. Letzig, J.A. Wollmershauser, S.R. Agnew, Metall. Mater. Trans. A 43, 1347 (2012).

[8] N. Stanford, M.R. Barnett, Mater. Sci. Eng. A 496 (2008).

[9] T. Al-Samman, X. Li, Mater. Sci. Eng. A 528, 3809 (2011).

[10] N. Stanford, Mater. Sci. Eng. A 565, 469 (2013).

[11] S. Gall, S. Müller, W. Reimers, Int. J. Mater. Form. 6, 187 (2013) 
[12] J. Bohlen, O. Schlung, S. Gall, S. Müller, D. Letzig, in: Magnesium Technology 2016, Proceedings of TMS, Eds. K.N. Solanki, D. Orlov, A. Singh, N.R. Neelameggham, 2016, p. 251.

[13] J. Bohlen, S. Yi, D. Letzig, K.U. Kainer, Mater. Sci. Eng. A 527, 7092 (2010).

[14] L. Nascimento, S.Yi, J.Bohlen, L. Fuskova, D. Letzig, K.U. Kainer, Proc. Eng. 2, 743 (2010).

[15] V. Kree, J. Bohlen, D. Letzig, K.U. Kainer, Prakt. Metallogr. 41, 233 (2004).

[16] F. Bachmann, R. Hielscher, H. Schaeben, Sol. St. Phen. 160, 63 (2010).
[17] I.L. Dillamore, W.T. Roberts, Metall. Rev. 10, 271 (1965).

[18] J. Bohlen, S.B. Yi, J. Swiostek, D. Letzig, H.G. Brokmeier, K.U. Kainer, Scr. Mater. 52, 259 (2005).

[19] S. Yi, H.G. Brokmeier, D. Letzig, J. Alloys Compound. 506, 364 (2010).

[20] J.P. Hadorn, R.P. Mulay, K. Hantzsche, S. Yi, J. Bohlen, D. Letzig, S.R. Agnew, Metall. Mater. Trans. A 44, 1566 (2013). 